\title{
Commentary: Dark matter and the missing lnc
}

\author{
Peter H. U. Lee, MD, PhD, MPH, and Nahush A. Mokadam, MD, FACS, FACC
}

\author{
From the Division of Cardiac Surgery, The Ohio State University Wexner Medical Center, Columbus, Ohio. \\ Disclosures: N.A.M. serves as a consultant and investigator for Abbott, Medtronic, and SynCardia. P.H.U.L. has \\ nothing to disclose with regard to commercial support. \\ Received for publication Jan 30, 2019; accepted for publication Jan 31, 2019; available ahead of print Feb 28, \\ 2019. \\ Address for reprints: Nahush A. Mokadam, MD, FACS, FACC, Division of Cardiac Surgery, Kakos and Williams \\ Endowed Professor in Cardiac Surgery, N-825 Doan Hall, 410 W 10th Ave, Columbus, OH 43210 (E-mail: \\ Nahush.Mokadam@osumc.edu). \\ J Thorac Cardiovasc Surg 2020;159:61 \\ $0022-5223 / \$ 36.00$ \\ Copyright (C) 2019 by The American Association for Thoracic Surgery \\ https://doi.org/10.1016/j.jtcvs.2019.01.114
}

The renowned late theoretical physicist Stephen Hawking once said that "the missing link in cosmology is the nature of dark matter and dark energy." For centuries, space was believed to be, as the name implies, empty and void. It is now hypothesized, however, that approximately $95 \%$ of the universe is made up of dark matter and dark energy. ${ }^{1}$ And although these mysterious forms of matter and energy are invisible and essentially undetectable, they both are presumed to exist and have a profound effect on the nature of galaxies and the universe. Similarly, the dogma that DNA is transcribed into messenger RNA, which is then translated into peptides and proteins, has historically dominated our understanding. Or so we thought. The surprising discovery of the noncoding RNAs, and more specifically long noncoding RNA (lncRNA), has revolutionized our understanding of the critical role these seemingly unimportant strands of RNA have in the essential function of many, if not most, biologic processes, including neurological injury. ${ }^{2}$ And as we learn more about the ubiquitous nature of lncRNAs, their potential clinical value as a biomarker or therapeutic target or agent for diagnosing and treating a variety clinical conditions is becoming increasingly evident.

In this issue of the Journal, Gao and colleagues ${ }^{4}$ describe an elegant study that demonstrates for the first time the potential role of a lncRNA (GAS5) in mediating neurologic injury caused by deep hypothermic circulatory arrest (DHCA). Gao and colleagues ${ }^{4}$ demonstrated that downregulation of GAS5 reduced neuronal damage and neurologic function in mice after DHCA. Despite the inherent limitations of rodent experiments and of extrapolating these results to the clinical setting, this study does provide some clear and promising evidence of the potential role of a lncRNA in mediating neurologic injury after DHCA. Most significantly, the putative cerebral protection seen with GAS5 inhibition points to the potential utility of this and other lncRNAs as both powerful biomarkers and therapeutic targets for neuroprotection after DHCA.

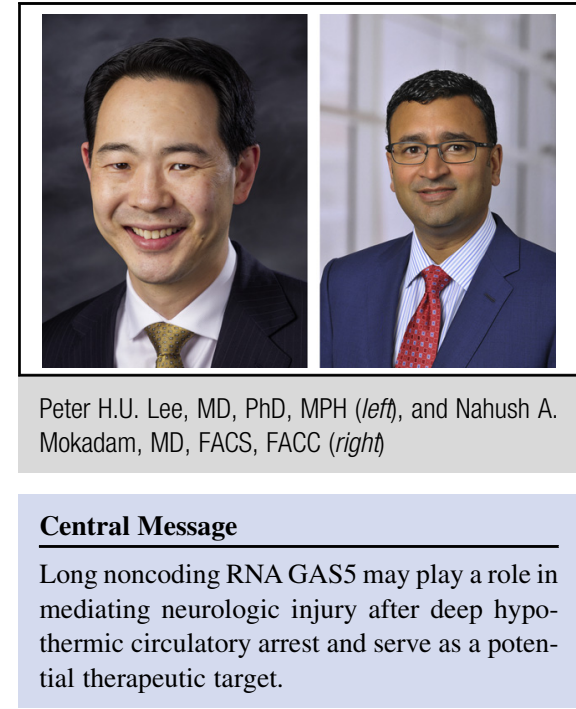

See Article page 50.
Neurologic injury remains the Achilles' heel of DHCA, and this study represents a clear demonstration of mitigation of downstream injury after DHCA. And although multiple pathways are involved, in this case, this lncRNA may indeed be a missing link between DHCA and neurologic injury. Just as the existence and critical role of dark matter and dark energy in the universe are becoming increasingly evident, despite still being largely an enigma, we are also just starting to appreciate better the critical function that noncoding RNAs once considered unimportant may have in mediating significant clinical problems. In the words of British novelist Matt Haig, "Dark matter is needed to hold galaxies together. Your mind is a galaxy." 5 Perhaps, in our effort to keep our mind together after DHCA, we have found our missing lnc!

\section{References}

1. Bahcall NA. Dark matter universe. Proc Natl Acad Sci U S A. 2015;112:12243-5.

2. Kaur H, Sarmah D, Saraf J, Vats K, Kalia K, Borah A, et al. Noncoding RNAs in ischemic stroke: time to translate. Ann N Y Acad Sci. 2018;1421:19-36.

3. Chen Y, Zhou J. LncRNAs: macromolecules with big roles in neurobiology and neurological diseases. Metab Brain Dis. 2017;32:281-91.

4. Gao S, Gu T, Shi E, Tang R, Liu J, Shi J. Inhibition of long noncoding RNA growth arrest-specific 5 attenuates cerebral injury induced by deep hypothermic circulatory arrest in rats. $J$ Thorac Cardiovasc Surg. 2020;159:50-9.

5. Haig M. The humans: a novel. New York: Simon \& Schuster; 2013. 